\title{
Bio-inspired Strategy for Control of Viral Spreading in Networks
}

\author{
Chinwendu Enyioha \\ Department of Electrical and \\ Systems Engineering \\ University of Pennsylvania \\ Philadelphia, PA 19104 \\ cenyioha@seas.upenn.edu
}

\author{
Victor Preciado \\ Department of Electrical and \\ Systems Engineering \\ University of Pennsylvania \\ Philadelphia, PA 19104 \\ preciado@seas.upenn.edu
}

\author{
George Pappas \\ Department of Electrical and \\ Systems Engineering \\ University of Pennsylvania \\ Philadelphia, PA 19104 \\ pappasg@seas.upenn.edu
}

\begin{abstract}
We consider a variant of the well-known Susceptible-InfectedSusceptible (SIS) network spreading model, and present a virus control strategy in which nodes in a network are in sleep state or awake state with certain probabilities. Nodes in sleep state are assumed to have a lower infection rate relative to nodes in awake state, hence lower exposure levels to a viral attack on the network. The strategy presented is inspired by the notion of bacteria colony persistence to antibiotics in which certain bacteria in the colony hibernate or switch to dormant states as a way of reducing their exposure to antibiotics and helping the colony withstand the effects of the antibiotic attack. Based on a simplified model of persistence, we present a threshold above which a small infection may become an epidemic. Further, we consider the problem of designing the probability of each node being in sleep (less infectious) state with the least effort, allowing the network to control the spread of an infection. Our design strategy for the probabilities of being in sleep state exploits the diagonal dominance property of a non-convex constraint, which enables relaxation of the problem to a Linear Program, for which we compute an exact solution using only local information. Finally, via simulations, we show that the probability of being in sleep state, resulting from our relaxation does, indeed, exploit the network structure in controlling the virus spread.
\end{abstract}

\section{Categories and Subject Descriptors}

D.4.6 [Security and Protection]: Optimization, Networks and Graphs

\section{Keywords}

Virus propagation; Convex optimization; Network assortativity.

\footnotetext{
* This work was supported by ONR-MURI HUNT award N00014-08-1-0696.
}

Permission to make digital or hard copies of all or part of this work for personal or classroom use is granted without fee provided that copies are not made or distributed for profit or commercial advantage and that copies bear this notice and the full citation on the first page. To copy otherwise, to republish, to post on servers or to redistribute to lists, requires prior specific permission and/or a fee.

HiCoNS'13, April 9-11, 2013, Philadelphia, Pennsylvania, USA

Copyright 2013 ACM 978-1-4503-1961-4/13/04 ...\$15.00.

\section{INTRODUCTION}

As networks become ubiquitous they also become prone to strategic attacks aimed at causing widespread damage in minimal time. A malicious intruder can, for instance, manipulate or infect one node in the network and have the infection spread to other nodes over time. Occurrence of such attacks are pervasive; examples of this infection spread can be found in computer networks [1], wireless devices, or human populations. Modelling spreading processes in networks is an important topic in epidemiology and has recently attracted substantial research attention. Several deterministic and stochastic models have been proposed to model the spread of biological diseases. A literature review on modelling of epidemics in human populations can be found in [2]. An important concept in mathematical analyses of epidemiological models is the existence of a threshold beyond which a small infection can result in an epidemic. Most recent models represent individuals as nodes or agents that interact in a contact network represented by a graph. In these graph-based models, the epidemic threshold is equal to the inverse of the spectral radius of the contact graph [3, $4,5]$.

We investigate how epidemic models can be used to design switching strategies to boost the resilience of the agent population against a contagion. The usual recourse to tackle this problem is to consider immunizing or isolating infected infected nodes. In computer networks, for instance, a solution to the spread of viruses is to install an anti-virus software to clean out the infected computers before the attack spreads to other computer systems on the network. While this approach may work, resources available for anti-viruses or other forms of immunization are usually limited. Determining minimal-cost ways to control and minimize the spread of such network attacks becomes imperative. Though some work has been done on the problem of controlling infection spread in networks, [6, 7]; the approaches presented tend to address the problem from the angle of expending some limited immunization resource.

As an alternative to existing works, we propose a bioinspired strategy to address the problem of controlling a viral spread in a network. The method we present is inspired by bacteria reaction to antibiotics. In particular, our work is inspired by a contagion mitigation strategy well studied in bacteria colonies termed persistence (we refer readers to $[8,9,10]$ for more information on this behavior). In bacteria colonies, a small portion of the population go into sleep or dormant states, thereby significantly reducing their exposure 
to attack (by the antibiotic); thus, strengthening the ability of the colony to survive the antibiotic attack and avoid extinction. A more recent work in the control theory research community studying this bacterial phenomenon appears in [11]; where the authors develop a population dynamic model and design methods to minimize the fraction of the network that transition into long-term dormancy using tools from optimal control. Attempts have also been made to link this trait, observed in bacteria, to strategies in human adaptation and survival [12].

In this work, we mathematically formalize a notion of persistence in a networked setting, and study the problem of determining the optimal probabilities of nodes to be in sleep state to prevent a small infection from resulting in an epidemic in the network. We present this strategy as a tool that can be used to defend networks against a viral attack. We employ well-studied, epidemiology-based models to describe a virus spread in a network $[13,14,15]$, with a goal of studying the interplay between the probabilities of being in either sleep or awake state and our adapted model of a virus propagation rate from [3]. For our model, we derive a tipping point, by presenting conditions under which the likelihood of infection at each node converges to zero. Furthermore, we consider the problem of optimally designing the probabilities of being in sleep state for the nodes and formulate the problem via a principled convex programming.

The organization of the paper is as follows: In section 2, we briefly review the SIS spreading model, our adapted SIS model and state our problems. Section 3 comprises results on stability of the model presented in 2 . We discuss the design of sleep probabilities in section 4, following up with simulations to validate our results in Section 5. In section 6 , we introduce assortativity and an important notion of centrality measure in communication networks - betweenness centrality $[16,17]$. We numerically show that network structural properties does affect the distribution of protection resources - probabilities of being in sleep mode to prevent an epidemic. In particular, we find that networks with highly assortative mixing patterns have low correlation between distribution of sleep probabilities and the betweenness centrality measure. Concluding remarks follow in Section 7.

\section{PROBLEM FORMULATION}

In this section, we briefly review the SIS model developed in [3]. We then introduce the SIS model with sleep and awake states as the basis for our developments. First, we introduce some graph-theoretical nomenclature.

Graph theory is the primary mathematical tool to represent the contact topology in an epidemic network. Let $G=$ $(V, E)$ represent a directed graph, with $V=\{1, \ldots, n\}$ being the set of nodes, and $E \subseteq V \times V$ being the set of edges. In our model, every agent is represented by a node. We assume that $G$ is a simple graph with unweighted, undirected edges and no self-loops. We denote by $N_{i}=\{j \in V:\{i, j\} \in E\}$ the neighborhood set of node $i$. We can represent the graph structure using the adjacency matrix $A=\left[a_{i j}\right] \in \mathbb{R}^{n \times n}$, where $a_{i j}=1$ if and only if $\{i, j\} \in E$, otherwise $a_{i j}=0$. The largest magnitude of the eigenvalues of the adjacency matrix $A$ is called the spectral radius of $A$ and is denoted by $\rho(A)$.

\subsection{Standard SIS Model}

A set of difference equations was derived in [3], representing the time evolution of the probability of infection for each individual in a network of agents following the SIS epidemic model. In this model, a network of $n$ individuals is considered where each individual is represented by a node and the contact topology is represented by a graph $G$. A disease in this model is characterized by infection rate $\beta \in \mathbb{R}_{+}$ and curing rate $\delta \in \mathbb{R}_{+}$. The SIS model describes the time evolution of the infection probability of the $i$-th individual, denoted by $p_{i} \in[0,1]$, as

$$
p_{i}(t+1)=1-\prod_{j \in N_{i}}\left(1-\beta p_{j}(t)\right)-\delta p_{i}(t) .
$$

After linearizing the above nonlinear, discrete-time model, [3] obtained the following result:

THEOREM 1. If an epidemic dies out, then it is necessarily true that $\rho(A)<\delta / \beta$, where $\beta$ is the birth rate, $\delta$ is the curing rate and $\rho(A)$ is the largest eigenvalue of the adjacency matrix $A$.

This result provides a simple epidemic threshold for arbitrary graphs, such that if the threshold condition is satisfied the epidemic dies out. In the following, we obtain a similar result for the case in which we allow nodes to randomly switch between two states: sleep and awake.

\subsection{Hybrid SIS Model}

In our model, we assume that each node (regardless of their infection level) or likelihood of infection, can be in one of three states - awake, sleep or infected. Our model is an extension of the traditional SIS model. In particular, we split the susceptible state into two different classes of susceptibility - sleep and awake. These two states are qualitatively similar to the susceptible state in the SIS model and their only difference is in the probabilities of infection. All nodes in the sleep state have an infection rate $\beta_{s}$ and a node in the awake state have a higher infection rate $\beta_{a}>\beta_{s}$. We also assume that all nodes in the network, independent of which state they are in, have a common virus curing rate $\delta$. The curing rate, $\delta$, can be interpreted as a degree of 'natural immunity' that each node in the network has, irrespective of its state.

Our model assumes that the probability of being in sleep state for node $i$ is $l_{i}$ (and the probability of being in awake state is $\left.1-l_{i}\right)$. At each time step, each node randomly chooses to be awake or asleep, independent of its previous state and the state of its neighbors.

This implies that the infection rate of node $i$ at time $t$, denoted by $b_{i}(t)$, is a random variable which is equal to $\beta_{s}$ with probability $l_{i}$, and is equal to $\beta_{a}$ with probability $1-l_{i}$. Assuming a discrete-time set-up, at each time-step $t$, each node $i$ has a probability $p_{i}(t)$ of being infected at time $t$. The evolution of the marginal probability of infection can be exactly described via a Markov chain with $3^{n}$ states, which in general is extremely large to be of practical interest. Following the mean-field approach proposed in [3], we can approximate the dynamics of the Markov Chain that describes the evolution of the probability of infection at each node. Furthermore, we can linearize the mean-field dynamics around the infection-free state. Following these steps, the evolution of the probability of infection for node $i$ is given by 


$$
\begin{aligned}
p_{i}(t+1) & =l_{i} \beta_{s} \sum_{j \in N(i)} a_{i j} p_{j}(t) \\
& +\left(1-l_{i}\right) \beta_{a} \sum_{j \in N(i)} a_{i j} p_{j}(t)+(1-\delta) p_{i}(t),
\end{aligned}
$$

which, defining $p(t)=\left(p_{1}(t), \ldots, p_{N}(t)\right)^{T}$, can be written in matrix-vector form as

$$
p(t+1)=B A p(t)+(1-\delta) \operatorname{Ip}(t),
$$

where the matrix $A$ is the adjacency matrix of the network and $B$ is the diagonal matrix $B=\operatorname{diag}\left(\beta_{s} l_{i}+\beta_{a}\left(1-l_{i}\right)\right)$. We denote by $L$ the diagonal matrix $L=\operatorname{diag}\left(l_{i}\right)$.

The first part of our work considers a case where all nodes in the network have a homogeneous probability of being in sleep state; that is, $l_{i}=l$. The later part of our analyses considers a heterogeneous model where each node $i$ has a certain probability of being in sleep rate $l_{i}$, which results in a particular profile for the infection rate $b_{i}=\beta_{s} l_{i}+\beta_{a}\left(1-l_{i}\right)$. Based on (2), next, we state the specific problems addressed in this paper.

\subsection{Problem Statements}

Given the above model and evolution of probability of infection in (2), we present the problems considered in this work.

Problem 1. (Epidemic Threshold) Given a network in which nodes have a probability of infection evolving according to (2), what is the point beyond which an infection on one node can propagate and infect other nodes resulting in an epidemic in the network?

Problem 2. (Homogeneous Design) Under the assumption that all nodes have a common probability of being in sleep state; that is, $l_{i}=l \forall i$, what should the optimal probability of being in sleep state be to prevent a virus infection on one node from resulting in an epidemic?

Problem 3. (Heterogeneous Design) Let each node $i$ have a distinct probability, $l_{i}$, of being in sleep state. What is the optimal $l_{i}$ to prevent an epidemic?

To approach the last problem, we derive near-optimal probabilities of being in sleep state via a convex relaxation. These problems are discussed in Sections 3 and 4.

\section{STABILITY ANALYSIS}

In this section, we consider the stability of (2) when the nodes in the network have a common probability of being in sleep mode. Following an approach similar to the one proposed in [3], we obtain the following result:

ThEOREM 2. Given the discrete-time linear system (2), and any initial condition $p(0)$, the probability of infection $p(t)$ converges to 0 , as $t \rightarrow \infty$ if and only if

$$
\rho(A)<\frac{\delta}{l \beta_{s}+(1-l) \beta_{a}} .
$$

Proof. (Theorem 2) To study the stability of the linearized model in (2), we only need to locate the eigenvalues of the transition matrix $B A+(1-\delta) I$. It is easy to prove that the eigenvalues of this transition matrix are

$$
\lambda_{i}(B A+(1-\delta) I)=b_{i} \lambda_{i}(A)+1-\delta,
$$

where $B=\operatorname{diag}\left(b_{i}\right)$ with $b_{i}=b_{j}$, for all pairs $i, j$. Hence, for all the eigenvalues of the transition matrix to be in the unit circle, we need the condition in the statement of the theorem to hold.

For a network under a virus attack, assuming the infection rates for nodes in sleep state in any network varies from those in awake states, a conclusion we can draw from Theorem 2 is that with control over the probabilities of being in sleep or awake state, the network can protect itself from an attack by having some nodes switch to sleep states to prevent a small infection from resulting in an epidemic and destabilizing the network. Two notable things about this resilience strategy are that no assumptions on the network structure are made; second, it is a network protection method that requires no resources for protection - a passive protection strategy, since some nodes only need to be in sleep state to reduce exposure to a virus attack.

\section{DESIGN PROBLEM}

Having established the epidemic threshold, we now consider a design problem of determining optimal probability of being in sleep state to minimize the spectral radius of the system matrix in (2). We consider this problem in two cases:

1. Homogeneous probabilities - In this instance, all nodes have the same probability of being in sleep state, in which case the matrix $B$ can be expressed as $B=b I$.

2. Heterogeneous probabilities - Each node has a distinct probability of being in sleep state, in which case, $B=$ $\operatorname{diag}\left(b_{i}\right)$, for $i=1, \ldots, N$.

\subsection{Homogeneous Sleep Probability}

The goal here is to put only few nodes to sleep, by finding the minimum possible common probability of being in sleep state for all nodes, such that the probability of infection, $p(t)$ converges to 0 . In other words, we interpret the probability of being in sleep state as a cost, which we want to minimize. We formulate this as the following optimization problem -

$$
\begin{array}{cl}
\underset{l}{\operatorname{minimize}} & l \\
\text { subject to } & \left(l\left(\beta_{s}-\beta_{a}\right)+\beta_{a}\right) \rho(A)+1-\delta \leq 1, \\
& 0 \leq l \leq 1,
\end{array}
$$

which is equivalent to

$$
\begin{array}{cl}
\underset{l}{\operatorname{minimize}} & l \\
\text { subject to } & \left(l\left(\beta_{s}-\beta_{a}\right)+\beta_{a}\right) \rho(A) \leq \delta, \\
& 0 \leq l \leq 1 .
\end{array}
$$

As seen in (5), the problem of optimally designing a common sleep probability $l$, subject to stability constraint is a linear program.

Proposition 1. The linear optimization problem (5) has a unique optimal solution.

Proof. To prove this, note that though the feasible set is constrained, it is non-empty, and imposes a bound on the objective function. Recall that the epidemic threshold is $\rho(A)<\frac{\delta}{l\left(\beta_{s}-\beta_{a}\right)+\beta_{a}}$. To get the optimal $l$, which we denote as $l^{*}$, we solve (5) for the $l$ that makes the inequality binding. 
Doing so yields

$$
l \leq \frac{\left(\frac{\delta}{\rho(A)}-\beta_{a}\right)}{\beta_{s}-\beta_{a}} .
$$

In other words,

$$
l^{*}=\inf \left\{l \mid \rho(A) \leq \frac{\delta}{l\left(\beta_{s}-\beta_{a}\right)+\beta_{a}}\right\},
$$

which implies that if the common probability of being in sleep state is $l \geq l^{*}$, an epidemic is prevented; and if $l<l^{*}$, an epidemic results. We note that a trivial solution $l=0$ will be infeasible, since (4) needs to be satisfied.

\subsection{Heterogeneous Sleep Probabilities}

In this case, the nodes have distinct probabilities of being in sleep mode, $l_{i}$, which we want to minimize, subject to stability of (2). A related problem was addressed in [18], where the proposed virus taming strategy was to allocate resources in a way that equalizes the propagation impact of each network component. Here, we adopt a disciplined convex formulation of the problem and present a convex relaxation that efficiently, and using only local information, designs the probability of being in sleep state. Our solution in this heterogeneous case has a closed-form expression, and requires only local information. Recall that $B=\beta_{a}(I-L)+\beta_{s} L$, where $L=\operatorname{diag}\left(l_{1}, \ldots, l_{N}\right)$ is a diagonal matrix of the probabilities of being in sleep state. We formally state the optimization problem below:

$$
\begin{array}{ll}
\underset{l_{i}}{\operatorname{minimize}} & \sum_{i}^{N} l_{i} \\
\text { subject to } & \rho(B A) \leq \delta, \\
& B=\beta_{a}(I-L)+\beta_{s} L \\
& 0 \preceq L \preceq I .
\end{array}
$$

Since $B$ is a function of $L$ and $\beta_{s}<\beta_{a}$, we reformulate (6) in terms of $B$, and make $B$ the optimization variable. If we apply Perron-Frobenius Theorem to the spectral radius constraint in (6), the problem is equivalent to

$$
\begin{array}{cl}
\underset{B}{\operatorname{maximize}} & \text { Trace } B \\
\text { subject to } & \delta B^{-1}-A \succeq 0 \\
& \beta_{s} I \leq B \leq \beta_{a} I .
\end{array}
$$

First, we note that though problem (7) has a linear cost function, it is not a convex program because of the negative exponent in the first summand of the Semidefinite constraint. To address the challenge of nonconvexity from the Semidefinite constraint, we shall use a sufficient condition on diagonally dominant matrices, and eventually arrive at an elegant linear program.

Definition 1. A matrix is said to be diagonally dominant if for every row of the matrix, the magnitude of the diagonal entry in each row is larger than or equal to the sum of the magnitudes of the off-diagonal entries of that row. More formally, the matrix $Z$ is diagonally dominant if

$$
\left|z_{i i}\right| \geq \sum_{j \neq i} z_{i j} \forall i
$$

LEMma 1. A diagonally dominant symmetric matrix with non-negative diagonal entries is positive semidefinite.
See [19] for a proof of Lemma 1. Because we do not allow for self-loops in the network, the diagonal entries of the network adjacency matrix $A$ are 0 , and the sum of the off-diagonal entries of row $i$ is the degree of node $i$ in the network, which we denote as $\operatorname{deg}(i)$. In scalar form, we can express the Semidefinite constraint $\delta B^{-1}-A \succeq 0$ as $\frac{\delta}{b_{i}}-\operatorname{deg}(i) \geq 0$, for each node $i$, where $b_{i}$ is the $i$ th element of the diagonal matrix $B$ in (7), and $\operatorname{deg}(i)$ denotes the degree of node $i$. We can express the constraint $\frac{\delta}{b_{i}}-\operatorname{deg}(i) \geq 0$ as

$$
b_{i} \leq \frac{\delta}{\operatorname{deg}(i)} .
$$

In light of this relaxation, we reformulate problem (7) as an linear program as follows

$$
\begin{array}{ll}
\underset{b_{i}}{\operatorname{maximize}} & \sum_{i=1}^{N} b_{i} \\
\text { subject to } & b_{i} \leq \frac{\delta}{\operatorname{deg}(i)} \\
& \beta_{s} \leq b_{i} \leq \beta_{a},
\end{array}
$$

from which we derive the closed-form solution

$$
b_{i}=\min \left\{\beta_{a}, \frac{\delta}{\operatorname{deg}(i)}\right\} .
$$

After deriving $b_{i}$ for node $i$ in (10), it is trivial to solve for the probability of being in sleep state $l_{i}$.

REMARK 1. It is remarkable that the solution obtained for the sleep probabilities in the heterogeneous case depend only on local information - individual node degrees. This means that the probability of being in sleep state for each node, can be computed independent of other nodes and complete knowledge of the network. We note that this solution is particularly applicable to large networks where significant costs can be incurred by centrally determining a policy for the probability of being in sleep state and communicating it to the nodes.

\section{SIMULATIONS AND DISCUSSIONS}

\subsection{Simulations Parameters}

In this section, we present results of experiments, indicating how the probabilities of being in sleep states $l_{i}$ is distributed across the nodes in the network. We solve the problem for a small world network model, and Erdos-Renyi network model as well as networks with homogeneous degrees. For the experiments, we consider a network of size $n=300$, using a fixed curing rate of $\delta=0.04$, sleep state infection rate of $\beta_{s}=0.5 \frac{\delta}{\rho(A)}$ and awake state infection rate of $\beta_{a}=1.5 \frac{\delta}{\rho(A)}$. In the simulations, we compare how the probabilities of being in sleep state vary with common network centrality measures including eigenvector, betweenness and degree centralities.

\subsection{Homogeneous Degree Network}

For a network with homogeneous node degrees, we can see in Figure 1 that the optimal probability of being in sleep state to prevent an epidemic is uniform across all nodes in the network, as expected. 


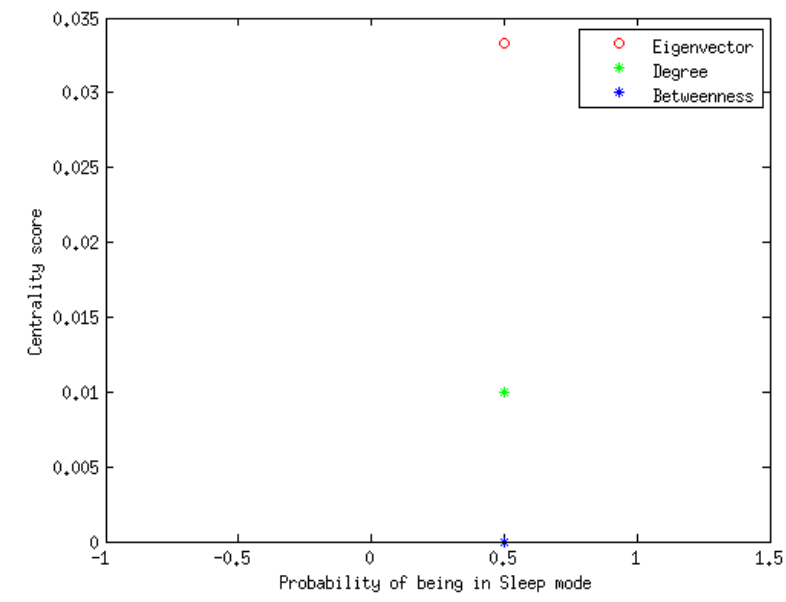

Figure 1: Figure showing sleep probabilities for a complete network

\subsection{Erdos-Renyi Network}

For an Erdos-Renyi graph with a link probability of 0.13 , having a degree distribution that is approximately Poisson, we see that the optimal solution is to allocate higher sleep probabilities to high degree nodes, establishing a largely positive correlation between the sleep probabilities and different network centrality measures, as can be seen in Figure 2 .

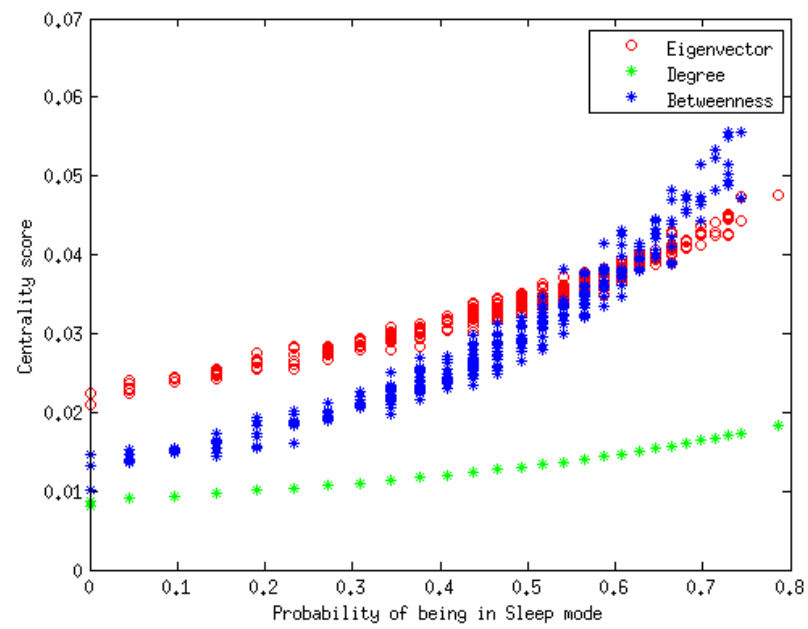

Figure 2: Figure showing sleep probabilities for nodes in an Erdos-Renyi network

\subsection{Small-World Network}

Finally, we implement our result on a small world network comprising $N=300$ nodes with probability 0.1 of rewiring each edge. In the small world network, a positive correlation between the probabilities of being in sleep mode and different network centrality measures is also observed. This is captured in Figure 3.

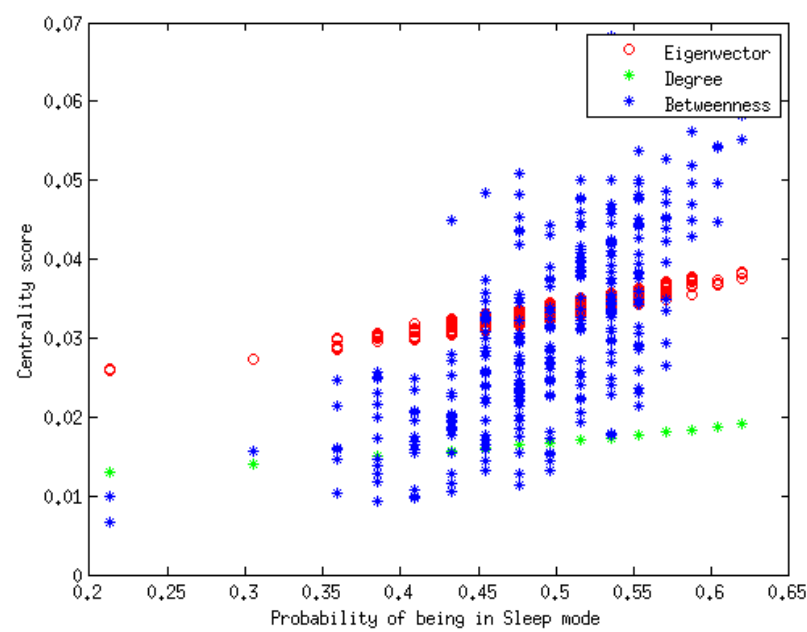

Figure 3: Figure showing sleep probabilities for nodes in a small world network

\section{EFFECTS OF NETWORK STRUCTURE}

In this section, we briefly introduce assortative mixing patterns in networks, eigenvector and betweenness centrality measures to demonstrate the effect of the network structure on the distribution of the sleep probabilities. The aim of this section is to numerically highlight how the network structure affects the distribution of the sleep probabilities.

\subsection{Assortativity in Networks}

Assortativity is a property of networks that explains its mixing pattern - the tendency for a node to donnect primarily with other nodes of similar degree. Examples of networks exhibiting this property include social networks $[20,21]$ where people who have similar tastes or interests are more likely to connect and establish relationships with one another. Disassortativity explains the converse concept where nodes with high degrees connect to low degree nodes. Assortativity and disassortativity are typically used to explain structural properties in networks and quantify the likelihood for preferential association within the network [22]. More formally,

DEFINITION 2. The (un-normalized) assortativity of a network, can be defined as

$$
R=\sum_{1 \leq\{i, j\} \leq N} d_{i} d_{j},
$$

where $d_{i}$ and $d_{j}$ are the respective degrees of nodes $i$ and $j$.

\subsection{Centrality}

Centrality measures in networks are metrics used to capture the level of importance of a given node relative to other nodes in the networks. ${ }^{1}$ Here, we focus on betweenness centrality, partly because of its importance as an index in epidemiology and communication networks $[16,17]$.

\footnotetext{
${ }^{1}$ For more on centrality measures, we refer readers to [23].
} 


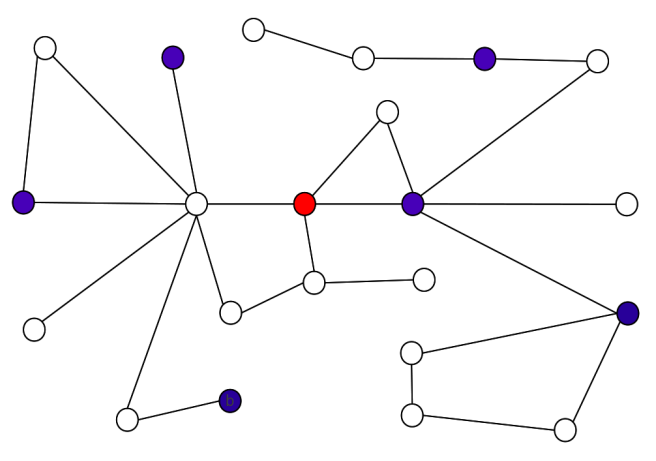

Figure 4: Network centrality depiction

\subsubsection{Betweenness Centrality}

It is a measure that indicates how relevant a node is in a network using shortest path routing. More formally, the betweenness centrality of a given node $i$ is

$$
B C(i)=\sum_{k \neq j: i \notin\{k, j\}} \frac{P_{i}(k, j) / P(k, j)}{(N-1)(N-2) / 2},
$$

where $P_{i}(k, j)$ denotes the number of geodesics (shortest paths) between nodes $k$ and $j$, that includes $i, P(k, j)$ is the total number of geodesics between $k$ and $j$. As an example, the red-colored node in Figure 4 has the highest betweenness centrality measure. ${ }^{2}$

\subsubsection{Eigenvector centrality}

Uses the principal eigenvector of the network adjacency matrix as a measure of importance in the network. More formally, suppose $A=\left[a_{i j}\right]$ is the adjacency matrix of the graph, and $a_{i j}=1$ if node $i$ is connected to node $j$, and $a_{i j}=0$, otherwise. The eigenvector centrality score $x_{i}$ of node $i$ is

$$
x_{i}=\frac{1}{\lambda} \sum_{j \in G} a_{i j} x_{j},
$$

where $\lambda$ is a proportionality factor, typically the principal eigenvalue.

\subsection{Graph Rewiring}

\subsection{Rewiring}

To modify the degree assortativity of the network, we carry out a degree-preserving rewiring, which has been studied in different contexts $[24,25]$. This rewiring can be described as follows: We pick two edges at random. Let's assume that those edges are $\{i, j\}$ and $\{r, s\}$. Notice that a rewiring comprising the removal of edges $\{i, j\},\{r, s\}$, while adding the edges $\{i, s\},\{r, j\}$ does preserve the degrees of nodes $i, j, r, s$. We can compute the effect of this rewiring on the assortativity as follows

$$
\triangle R_{i, j}^{r, s}=d_{i} d_{j}+d_{r} d_{s}-\left(d_{i} d_{s}+d_{r} d_{j}\right) .
$$

If $\triangle R_{i, j}^{r, s}$ is greater than 0 , the rewiring increases the assortativity of the network. On the other hand, if $\triangle R_{i, j}^{r, s}$ is lower

\footnotetext{
${ }^{2}$ Depending on a network attack objective, the red node is
} a target. than 0 , the network assortativity is reduced. We can sample through as many edges as we desire, using (12) to determine whether to rewire or not, to tune the assortativity of a network.

\subsubsection{Small World Network}

We generated a small world network via the following parameters $N=500$ nodes, with probability 0.15 of rewiring each edge. The assortativity coefficient was first -0.0083 . After allowing up to $10^{5}$ rewirings, the assortativity coefficient was increased to 0.8403 . Figure 5 shows the relation between the probabilities of being in sleep state and the centrality measures for the original and rewired network. As can be seen, the positive linear correlation between the sleep probabilities and the betweenness centrality measure drops from 0.8014 in the original network to 0.0709 in the rewired network with increased assortativity. And the correlation coefficient between the distribution of sleep probabilities and the eigenvector centrality measure changes from 0.8917 to 0.9428 .
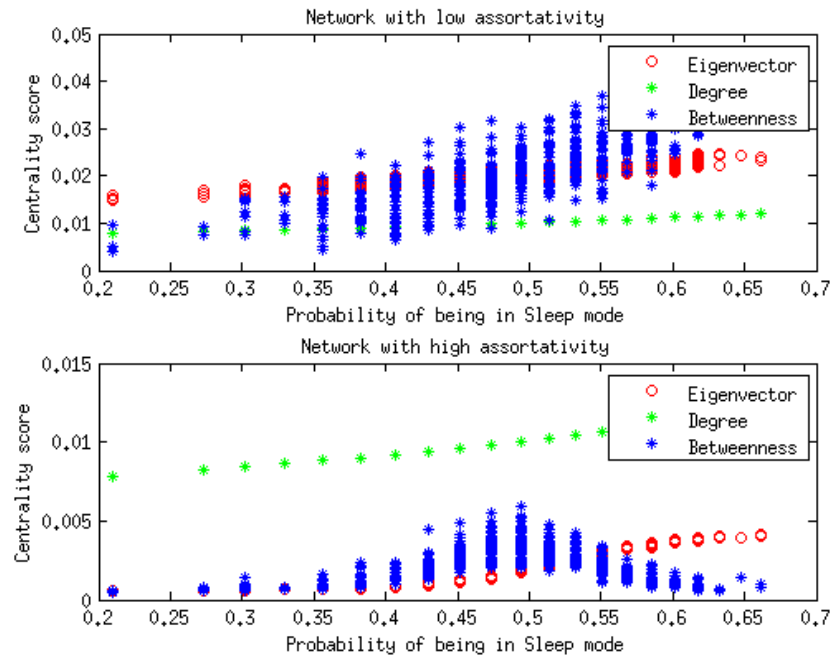

Figure 5: Comparing probabilities of being in sleep state with network Assortativity for a Small World network.

\subsubsection{Erdos-Renyi Network}

Here, we use $N=500$ nodes, with an edge probability of 0.13. The assortativity coefficient was first -0.0050 . After allowing up to $10^{5}$ rewirings, it was upped to 0.7535 . Via the same relaxation of the previous section, we were able to solve the problem for both the original and rewired networks. In Figure 6, the correlation coefficient between the distribution of sleep probabilities and the betweenness centrality measure changes significantly. In particular, it falls from 0.9619 for the original network to 0.0941 for the graph with increased assortativity. And the correlation coefficient between the distribution of sleep probabilities and the eigenvector centrality measure drops from 0.9818 to 0.9451 . Figures 5 and 6 indicate that the network structural property affects how the virus spread is controlled. 

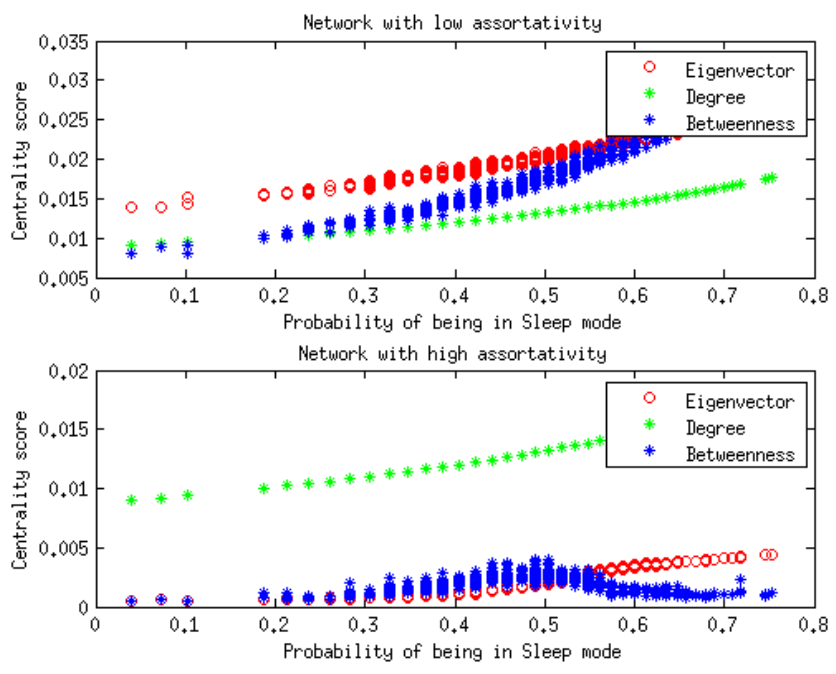

Figure 6: Comparing probabilities of being in sleep state with network Assortativity for an Erdos-Renyi network.

\section{CONCLUSIONS}

We presented a model of the concept of persistence (observed in bacteria colonies), in a networked setting and presented it as a tool that can be used to prevent a network attack from resulting in an epidemic. Based on our model of persistence, we derived a tipping point under which the probability of infection at each node of the network converges to zero. We also formulated the problem of designing optimal sleep probabilities for nodes in network to ensure an epidemic does not result when the network is under attack. Simulations based on the results from our relaxation showed a positive correlation between the probability of being in sleep state and different network centrality measures. Our simulations, in addition, show that the positive correlation holds for networks with neutral mixing patterns.

Some ongoing work include characterizing other convex approximations and heuristics for our heterogeneous optimization problem, in addition to computing bounds for our relaxation. In a related work, we are studying the problem of simultaneously controlling a contagion, while maximizing information flow in a communication network. We also plan to carry out analyses on the findings highlighted in the preceding section, as understanding the effects of network structural properties on distribution of protection resources can help in the design of resilient cyber-physical networks.

\section{ACKNOWLEDGEMENTS}

The authors gratefully acknowledge Michael Zargham and Ali Jadbabaie for their comments and suggestions on this work.

\section{REFERENCES}

[1] M.E.J. Newman, S. Forrest, and J. Balthrop. Email networks and the spread of computer viruses. Physical Review E, 66(3):035101, 2002.

[2] Norman TJ Bailey et al. The mathematical theory of infectious diseases and its applications. Charles Griffin
\& Company Ltd, 5a Crendon Street, High Wycombe, Bucks HP13 6LE., 1975.

[3] Y. Wang, D. Chakrabarti, C. Wang, and C. Faloutsos. Epidemic spreading in real networks: An eigenvalue viewpoint. In Reliable Distributed Systems, 2003. Proceedings. 22nd International Symposium on, pages 25-34. IEEE, 2003.

[4] Ayalvadi Ganesh, Laurent Massoulié, and Don Towsley. The effect of network topology on the spread of epidemics. In INFOCOM 2005. 24th Annual Joint Conference of the IEEE Computer and Communications Societies. Proceedings IEEE, volume 2, pages 1455-1466. IEEE, 2005.

[5] Piet Van Mieghem, Jasmina Omic, and Robert Kooij. Virus spread in networks. Networking, IEEE/ACM Transactions on, 17(1):1-14, 2009.

[6] R. Pastor-Satorras and A. Vespignani. Immunization of complex networks. Arxiv preprint cond-mat/0107066, 2001.

[7] Y. Chen, G. Paul, S. Havlin, F. Liljeros, and H.E. Stanley. Finding a better immunization strategy. Physical Review Letters, 101(5):58701, 2008.

[8] E. Rotem, A. Loinger, I. Ronin, I. Levin-Reisman, C. Gabay, N. Shoresh, O. Biham, and N.Q. Balaban. Regulation of phenotypic variability by a threshold-based mechanism underlies bacterial persistence. Proceedings of the National Academy of Sciences, 107(28):12541, 2010.

[9] N.Q. Balaban, J. Merrin, R. Chait, L. Kowalik, and S. Leibler. Bacterial persistence as a phenotypic switch. Science's STKE, 305(5690):1622, 2004.

[10] R.W. Kendall, C.P. Duncan, J.A. Smith, and J.H. Ngui-Yen. Persistence of bacteria on antibiotic loaded acrylic depots: a reason for caution. Clinical orthopaedics and related research, 329:273, 1996.

[11] N.G. Cooper and A.A. Julius. Bacterial persistence: Mathematical modeling and optimal treatment strategy. In American Control Conference (ACC), 2011, pages 3502-3507. IEEE, 2011.

[12] A. Gardner, S.A. West, and A.S. Griffin. Is bacterial persistence a social trait? PLoS One, 2(8):e752, 2007.

[13] J.C. Frauenthal and JC Frauenthal. Mathematical modeling in epidemiology. Springer-Verlag Berlim, 1980.

[14] J.O. Kephart and S.R. White. Directed-graph epidemiological models of computer viruses. In Research in Security and Privacy, 1991. Proceedings., 1991 IEEE Computer Society Symposium on, pages 343-359. IEEE, 1991.

[15] J.O. Kephart and S.R. White. Measuring and modeling computer virus prevalence. In Research in Security and Privacy, 1993. Proceedings., 1993 IEEE Computer Society Symposium on, pages 2-15. IEEE, 1993.

[16] K.A. Lehmann and M. Kaufmann. Decentralized algorithms for evaluating centrality in complex networks. WSI, 2003.

[17] A. Tizghadam and A. Leon-Garcia. Betweenness centrality and resistance distance in communication networks. Network, IEEE, 24(6):10-16, 2010.

[18] Y. Wan, S. Roy, and A. Saberi. Designing spatially 
heterogeneous strategies for control of virus spread. Systems Biology, IET, 2(4):184-201, 2008.

[19] Abraham Berman and Naomi Shaked-Monderer. Completely positive matrices. World Scientific Publishing Company Incorporated, 2003.

[20] M.E.J. Newman. Assortative mixing in networks. Physical Review Letters, 89(20):208701, 2002.

[21] M. Catanzaro, G. Caldarelli, and L. Pietronero. Assortative model for social networks. Physical Review E, 70(3):037101, 2004.

[22] M.E.J. Newman. Mixing patterns in networks. Physical Review E, 67(2):026126, 2003.
[23] M.O. Jackson. Social and economic networks. Princeton University Press, 2010.

[24] P. Van Mieghem, H. Wang, X. Ge, S. Tang, and FA Kuipers. Influence of assortativity and degree-preserving rewiring on the spectra of networks. The European Physical Journal B-Condensed Matter and Complex Systems, 76(4):643-652, 2010.

[25] C.K. Enyioha, D.C. Tarraf, L. Li, and J.C. Doyle. On the graph of trees. In Control Applications, $(C C A) \&$ Intelligent Control,(ISIC), 2009 IEEE, pages 246-248. IEEE, 2009. 